\title{
Transversus Abdominis Plane (TAP) and Rectus Sheath Blocks: a Technical Description and Evidence Review
}

\author{
Vishal Uppal $^{1} \cdot$ Sushil Sancheti ${ }^{2} \cdot$ Hari Kalagara $^{3}$
}

Published online: 25 October 2019

(C) The Author(s) 2019

\begin{abstract}
Purpose of Review This article aims to review the anatomy relevant to the ultrasound techniques of the transversus abdominis plane (TAP) block and rectus sheath block (RSB). We discuss their analgesic efficacy for various surgical procedures, both as single shot and as a continuous infusion via catheters.

Recent Findings RSB provides superior analgesia to local infiltration and has an opioid-sparing effect for umbilical surgery, laparotomy, and laparoscopic surgery. There is no high-quality evidence comparing RSB with epidural analgesia. Intermittent bolus through catheters appears to be more effective than continuous infusion for prolonged analgesia. Similarly, ultrasoundguided TAP block provides postoperative analgesic benefit after abdominal laparotomy or laparoscopy and cesarean delivery when long-acting neuraxial opioids are not used or contraindicated. Adding adjuvant such as dexamethasone and dexmedetomidine to local anesthetic prolongs the duration of TAP and RSB blocks and improves their efficacy.

Summary Use of ultrasound guidance makes the RSB and TAP blocks more reliable. Single-shot infiltration is useful for less extensive surgical procedures, while catheters are a useful alternative when thoracic epidural analgesia is contraindicated.
\end{abstract}

Keywords Rectus sheath block $\cdot$ Rectus sheath catheter $\cdot$ Abdominal wall block $\cdot$ Transversus abdominis plane block $\cdot$ Transversus abdominis plane catheter

\section{Anatomical Concepts}

The rectus abdominis muscle (RAM) is a paired vertical muscle separated in the midline by the linea alba, it is wide and thin superiorly, increasing in thickness inferiorly. This muscle is surrounded by the rectus sheath, which is comprised of the aponeurosis of the more lateral abdominal muscles. The posterior layer becomes thin at the arcuate

This article is part of the Topical Collection on Regional Anesthesia

Vishal Uppal

v.uppal@dal.ca

1 Department of Anesthesia, Perioperative Medicine and Pain Management, Nova Scotia Health Authority and IWK Health Centre, Dalhousie University, Halifax, Nova Scotia B3H 2Y9, Canada

2 Department of Anesthesia, Memorial University of Newfoundland, St. John's, Canada

3 Department of Anesthesiology \& Perioperative Medicine, The University of Alabama at Birmingham (UAB), Birmingham, AL, USA line, one-third of the way between the umbilicus and the pubic crest. At this line, the aponeurosis of the internal oblique and transversus abdominis goes anterior to the muscle, rather than posterior, leaving only the thin transversalis fascia posterior. The deep inferior and superior epigastric arteries run deep to rectus abdominis and superficial to the fibrous layer that forms the posterior rectus sheath. Care should be taken to avoid puncturing these blood vessels during the needle insertion.

Lateral to the RAM are the external oblique, internal oblique, and transversus abdominis muscles (superficial to deep). The intercostal nerves run in a plane between the internal oblique and transversus abdominis muscles, then penetrate the posterolateral border of the rectus muscle, innervating the muscle (muscular branch) and then the subcutaneous tissue and skin (anterior cutaneous branch). The deep circumflex iliac artery can be seen in the posterior TAP plane, where needle puncture can lead to hematoma.

Instillation of local anesthetic bilaterally between the rectus muscle and the posterior sheath provides midline analgesia for several dermatomes around the injection site. Injection between the internal oblique and transversus abdominis muscles 
(TAP block) at the level of the iliac crest can provide analgesia for lower abdominal surgery. Similarly, local anesthetic injection between the rectus abdominis muscle and transversus abdominis muscle at subcostal level (subcostal TAP block) can provide analgesia for upper abdominal surgery.

It is important to note that only the somatic components of pain are covered when these blocks are utilized (skin, subcutaneous tissue, muscle). A multimodal analgesics regimen is required to cover the visceral pain component. Furthermore, bilateral blocks are required for midline incisions.

\section{Rectus Sheath Block}

\section{Introduction}

Rectus sheath block (RSB) was originally described in 1899 and was used to provide abdominal wall relaxation during laparotomy before the introduction of neuromuscular blocking agents [1]. Currently the block is generally performed for postoperative analgesia, either as a single injection or with a continuous catheter technique. While the block was traditionally used for procedures around the umbilicus to provide analgesia from T9-T11, good clinical results are also achieved for higher dermatomes, up to T6, when the injection is performed higher. This makes the block useful for midline laparotomy, regardless of its level in the abdomen.

\section{Ultrasound-Guided Rectus Sheath Block (Fig. 1)}

Real-time ultrasound guidance is our preferred method for placing rectus sheath blocks as it ensures the local anesthetic is efficiently placed anterior to the posterior rectus sheath without being within the rectus muscle [2]. A linear array ultrasound transducer is placed on the abdomen in a transverse position congruent with the surgical incision (generally between T8 and T11 dermatomes). In the middle of the abdomen, the linea alba is observed on the ultrasound screen. The probe is then moved laterally to identify the rectus muscle, along with the subcutaneous tissue and the peritoneum.

At the lateral border of the rectus muscle, the external oblique, internal oblique, and transversus abdominus can be identified. The needle tip is placed just deep to the posterolateral border of the rectus muscle. Correct placement is confirmed when injection causes the rectus muscle to lift cleanly off the rectus sheath deep to it (Fig. 1). Continuous hydrodissection during needle advancement is invaluable for efficiently identifying the correct plane. Between 10 and $15 \mathrm{ml}$ of local anesthetic per side or $0.25 \mathrm{ml} / \mathrm{kg}$ in pediatrics is the usual dose. Another approach is to use a smaller dose at two different levels on each side when a large incision must be covered. Dexamethasone or dexmedetomidine can be added to the local anesthetic to prolong the block duration [3].

\section{Alternative Techniques for Rectus Sheath Block}

Landmark techniques can be utilized by either the anesthesiologist or the surgeon. A blunt bevel needle is advanced perpendicular to the abdominal wall at the lateral edge of the rectus muscle. After the skin is penetrated, the needle is withdrawn back to the skin then advanced through the first "pop" that represents the anterior rectus sheath. Next, the needle is advanced until it is pressed against the firm resistance of the posterior sheath. If performed by the surgeon during surgery with an open abdomen, the blunt needle can be palpated from within the abdomen. A correctly placed needle tip will have a distinctive "close, but not too close" feeling on palpation. Alternatively, there has also been a laparoscopically assisted approach described [4].

\section{Catheter Technique}

A catheter may be placed at the time of block performance to extend the duration of analgesia. Catheter regimens vary, but intermittent bolus seems to be more efficacious than continuous infusion [5]. For example, $10 \mathrm{ml}$ of $0.2 \%$ ropivacaine in each catheter every $4 \mathrm{~h}$ by a programmable pump is an efficacious and safe dose in adults.

\section{Analgesic Efficacy}

\section{Umbilical Hernia}

One recent randomized control trial (RCT) has compared RSB with placebo for umbilical hernia surgery in adults where they found lower 24-h opioid consumption (mean morphine consumption $3.73 \mathrm{mg}$ vs. $8.76 \mathrm{mg}$ ) and lower pain scores [6]. A meta-analysis of ten trials for RSB in pediatric umbilical surgery found a reduction in opioid use for the first $6-8 \mathrm{~h}(-0.03$ $\mathrm{mg} / \mathrm{kg})[7 \cdot \bullet]$.

\section{Laparotomy}

In laparotomy, RSB has been shown to significantly reduce morphine use $(0.7 \mathrm{mg}$ vs. $6.4 \mathrm{mg}$ ) [8] and pain scores (VAS score at 6 h, 2 vs. 3) when compared with placebo [9]. These studies either used a single-shot technique [8-11], combined with TAP block $[12,13]$, or with continuous catheters [13, 14•]. A retrospective case-control study suggested the analgesic efficacy of RSB for open pyloromyotomy in infants [15]. Some of the studies also found significant reductions in postoperative nausea/vomiting, sedation, and constipation, and improvements in patient satisfaction $[9,16]$.

Rectus sheath catheters have been compared with thoracic epidural analgesia (TEA). One retrospective observational study for pancreas transplant found there was no significant difference in rescue analgesia and the technique was preferred 


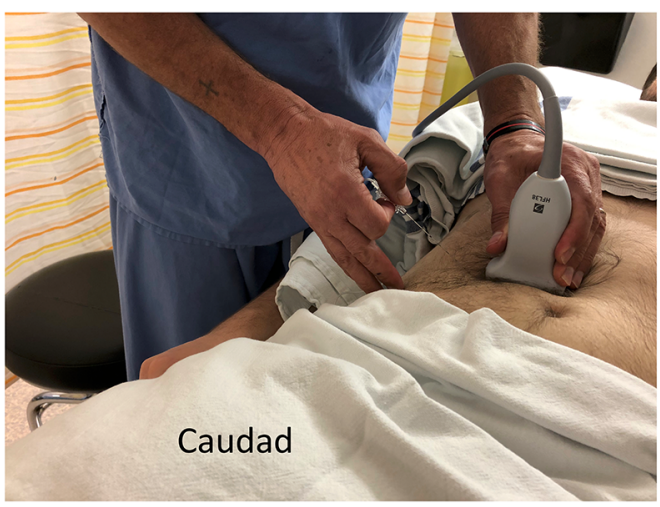

Fig. 1 Rectus sheath block. The left image shows the ultrasound probe position and in-plane needle insertion technique (lateral-to-medial approach) for a rectus sheath block (RSB). The image on right shows

due to their anticoagulation regimen [17]. Another observational study found equivalent pain scores and faster time to mobilization in the rectus sheath group [18].

\section{Laparoscopy}

Some recent studies have investigated the use of RSB for analgesia after laparoscopic surgery with positive results (24-h fentanyl use, 189 vs. $286 \mu \mathrm{g}$ [19]; and verbal and numeric pain score at $6 \mathrm{~h}, 3$ vs. 5 [20•]). In gynecological procedures, 2 RCTs showed RSB to be superior to placebo [19, 20•]. In one RCT of laparoscopic cholecystectomy, RSB resulted in less rescue analgesia compared with placebo and higher patient satisfaction compared with local infiltration [21]. For pediatric appendectomy, pain scores were reduced compared with placebo [22] and compared with in local infiltration [23] with similar overall [22] or reduced overall [23] opioid use.

\section{Complications}

Given the large area available for absorption after RSB, care should be taken to use appropriate doses and concentrations of local anesthetic [24••]. Adding epinephrine to reduce peak plasma concentration is also helpful [25].

The superior and inferior epigastric arteries are at risk of puncture or injury during the performance of rectus sheath block. Using ultrasound to avoid the arteries (and their attendant veins) can reduce the chance of puncture or inadvertent intravascular injection. When using a landmark technique, a lateral insertion point helps to avoid these vessels.

\section{Conclusion}

RSB has a proven analgesic benefit for umbilical surgery, laparoscopy, and laparotomy. It can provide midline abdominal analgesia anywhere from the symphysis pubis to

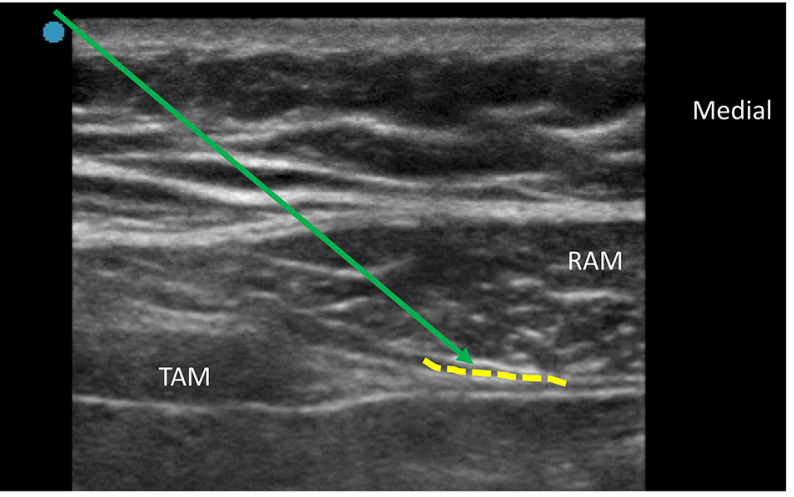

the sonogram during performance of RSB. RAM, rectus abdominis muscle; TAM, transversus abdominis muscle; green arrow, Needle path; yellow-shaded area denotes the site of local anesthetic (LA) deposition

the xiphoid process for several dermatomes around the injection point. RSB has few complications, which can be reduced by using ultrasound guidance. Single injection provides good analgesia for the first $6 \mathrm{~h}$, with a waning effect up to $24 \mathrm{~h}$. Continuous catheters can provide effective analgesia for extended durations.

\section{Tap Block}

\section{Introduction}

Rafi first described a technique of transversus abdominis plane (TAP) block using a landmark approach in 2001 . However, it was only after the introduction of the ultrasound-guided technique in 2007 that the TAP block was more widely adopted in clinical practice. In the past 20 years, it has been one of the most widely investigated regional anesthesia technique. Since the original description of ultrasound-guided technique, various technical modifications have been described including lateral [26], posterior [27], subcostal [28], and continuous catheter techniques [29] .

The exact technique used should be suited for a surgical incision, as each provides different dermatomal coverage. Lateral TAP block typically gives a T10-T12 dermatomal coverage while the posterior TAP approach covers T9-T12 and is shown to provide a longer duration of analgesia with possibly some visceral analgesia [30]. The subcostal TAP approach provides T6-T9 dermatomal coverage and is usually suitable for upper abdominal surgeries.

\section{Ultrasound-Guided TAP Block}

The ultrasound-guided techniques are recommended over the landmark technique to ensure precise deposition of 
local anesthetic between the transversus abdominis muscle and internal oblique muscle. Further, ultrasound may be helpful in reducing the risk of complications such as a vascular or visceral puncture.

\section{Lateral and Posterior TAP Block (Fig. 2)}

A linear high-frequency probe is generally ideal for performing the block. However, a curved array lower frequency probe may be needed in morbidly obese patients. After wide skin preparation with antiseptic, we recommend starting the scanning similar to the rectus sheath block (described earlier) to identify the rectus muscle. On sliding the ultrasound probe laterally towards iliac crest parallel to Tuffier's line, three lateral abdominal wall muscles (external oblique, internal oblique, and transverse abdominus muscle) can be easily visualized. Scanning more posteriorly the transversus abdominis muscles can be observed to taper. Since posterior TAP block is shown to provide longer analgesia and more extensive spread than lateral TAP block, posterior approach should be the preferred approach in clinical practice.

An 8-10-cm echogenic needle is inserted anterioposteriorly in-plane to ultrasound beam towards TAP plane (plane between transverse abdominus muscles and internal oblique). The main difference between the lateral and posterior TAP block is the final point of injection: mid-axillary line in lateral TAP block versus posterior axillary line for posterior TAP block. Hydrolocation is useful in determining that the needle tip is in correct plane; the clear separation of transversus abdominis muscle from internal oblique on injection is a good endpoint. At least 15$20 \mathrm{ml}$ of local anesthetic is required to produce a successful block in adult patients.

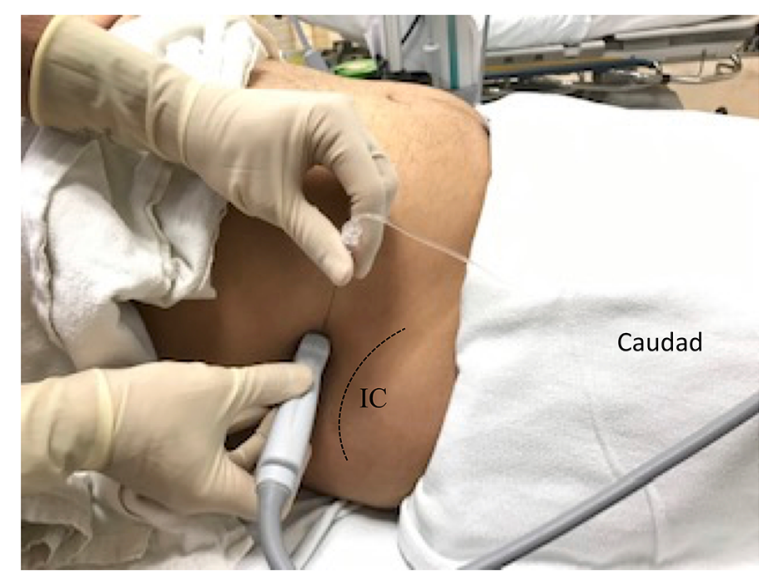

Fig. 2 Posterior transversus abdominis plane (TAP) block. The left image shows the ultrasound probe and in-plane needle insertion technique (medial-to-lateral or anterior-to-posterior approach) for a posterior TAP block. The image on the right shows the sonogram during performance of

\section{Subcostal TAP Block (Fig. 3)}

As the name suggests, the ultrasound probe position in the approach is parallel to the subcostal margin. For identification of the TAP plane, a similar scanning approach is followed with identification of the rectus muscle. On sliding the ultrasound probe laterally, the transversus abdominis muscle is usually seen underlying the lateral part of rectus abdominis muscle. On further lateral scan, the three lateral abdominal wall muscles can be visualized.

The exact point of local anesthetic injection depends on the dermatomal coverage required. A more medial injection (between rectus muscle and transversus abdominis muscle) will produce analgesia of T6-T8 dermatomes. On the other hand, a more lateral injection between transverse abdominus muscles and internal oblique will more consistently result in T8-T10 dermatomal analgesia. Both medial-to-lateral and lateral-tomedial in-plane needling techniques have been described [31]. We prefer a lateral-to-medial approach as it is more ergonomic to perform the technique and catheter insertion is away from the surgical field.

Oblique subcostal TAP block has been described as a modification of the subcostal technique. In this technique, an extralong $(15-20 \mathrm{~cm})$ needle is used to inject local anesthetic in TAP plane from subcostal region to iliac crest with a potential to provide T6-L1 dermatomal coverage. The technique is difficult to perform, and therefore, has not gained popularity [28].

\section{Catheter Technique}

Continuous catheters have been described for all of the above techniques. The technique of catheter insertion involves hydrodissection of the planes. The number and site of catheters needed depend on the site and extent of surgical incision.

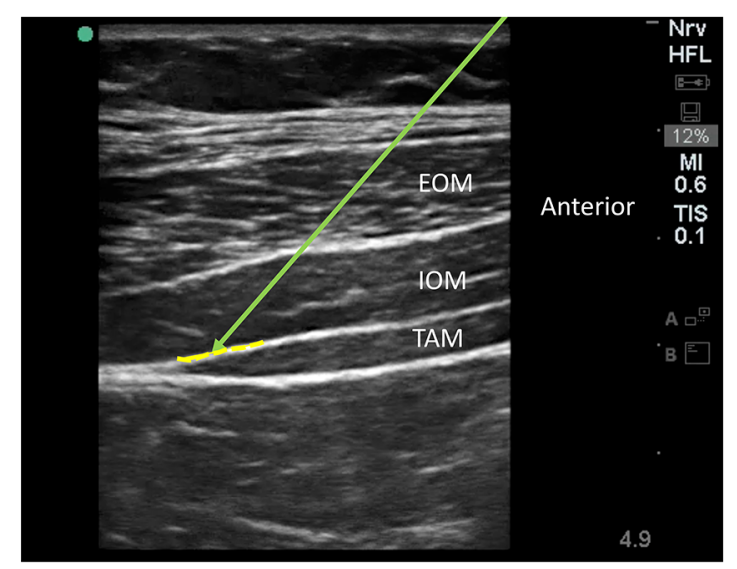

a posterior TAP block. EOM, external oblique muscle; IOM, internal oblique muscle; TAM, transversus abdominis muscle; green arrow, needle path; yellow-shaded area denotes the site of local anesthetic (LA) deposition 


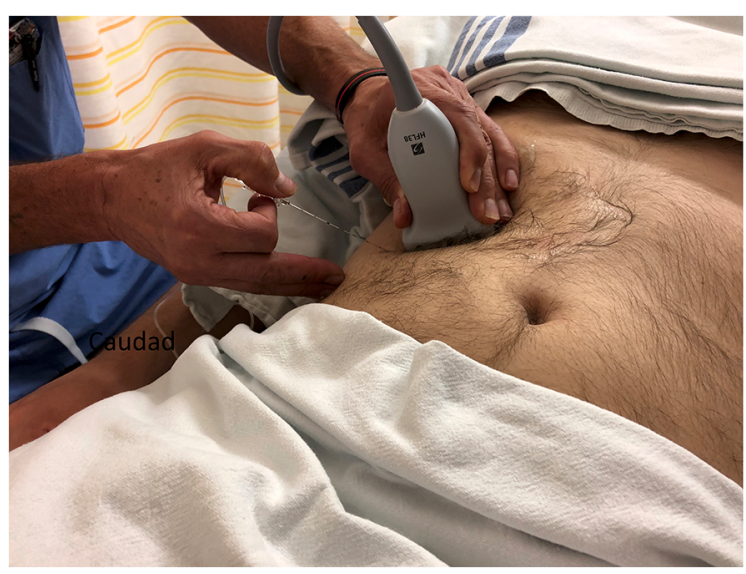

Fig. 3 Subcostal transversus abdominis plane (TAP) block. The left image shows the ultrasound probe position and in-plane needle insertion technique (lateral-to-medial or caudad-to-cephalad approach) for a subcostal TAP block. The image on the right shows the sonogram

Four-quadrant TAP catheters (bilateral subcostal and bilateral posterior TAP) can be used for midline abdominal incision extending from xiphisternum to pubic symphysis [29]. It must be stated that four catheters can be tedious to insert and maintain with further issues of local anesthetic systemic absorption and failure/dislodgement of one of the four catheters is high.

\section{Evidence Base}

A literature search of key databases for systematic reviews on TAP block for postoperative analgesia revealed more than 30 systematic reviews in different surgical populations. These include colorectal surgery, cesarean section, cholecystectomy, hysterectomy, appendectomy, donor nephrectomy, retropubic prostatectomy, and bariatric surgery.

\section{TAP Blocks Versus No Block/Placebo}

All recent reviews [32, 33・•, 34, 35] suggest that the ultrasound-guided TAP blocks reduce postoperative pain scores in the first 6-24 h postoperatively (mean difference of 1.4 to 2 points on a scale of $0-10)$ and reduce the morphine requirement in the first $24 \mathrm{~h}$ (mean difference $7-15 \mathrm{mg}$ ) compared with placebo $[33 \bullet \cdot, 34]$. Further, TAP block may delay the time to first analgesic request (mean difference $2 \mathrm{~h}$ ) [32] and may reduce the incidence of nausea/vomiting $[36,37]$. However, heterogeneity of type of surgery, block approaches, drugs, doses, and volumes warrant that the findings should be interpreted with caution.

Multiple systematic reviews have confirmed the analgesic efficacy of TAP block for open and laparoscopic colorectal surgeries [38, 39, 40•, 41-43]. Similarly, ultrasound-guided TAP block has been found to be an effective analgesic intervention in patients undergoing laparoscopic cholecystectomy $[44,45]$ and hernia surgery [46]. TAP blocks have also been

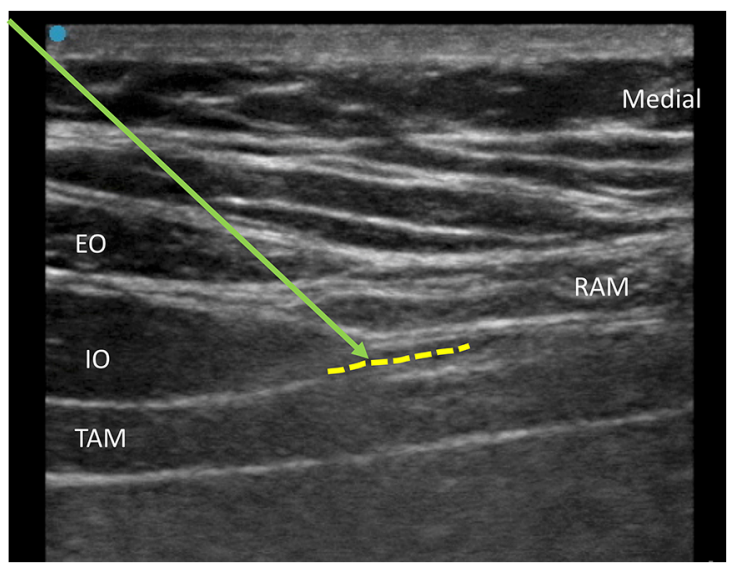

during performance of subcostal TAP block. RAM, rectus abdominis muscle; EO, external oblique; IO, internal oblique; TAM, transversus abdominis muscle; green arrow, needle path; yellow-shaded area denotes the site of local anesthetic (LA) deposition

shown to significantly lower intraoperative and cumulative postoperative 24-h opioid consumption, provide better pain control, and decrease the 24-h incidence of postoperative nausea vomiting for renal transplant recipients [47].

\section{TAP Blocks Versus Intrathecal Morphine}

TAP blocks do not appear to provide additional analgesic benefit in patients who also received intrathecal morphine [34]. Therefore, the TAP block may be particularly useful when neuraxial techniques or opioids are contraindicated. Some of the older reviews (conducted before 2012) found a limited benefit of TAP block in the context of multimodal analgesia [48-50].

\section{TAP Blocks Versus Thoracic Epidural Analgesia}

A recent review synthesized the data comparing TAP block with TEA. Pain scores at rest on postoperative day 1 were equivalent for TAP block and TEA groups in children and in adults. However, the TEA group experienced a higher rate of hypotension and longer hospital length of stay compared with the TAP block group, without a difference in functional outcomes [51•].

\section{TAP Block Versus Local Anesthetic Wound Infiltration}

Three different reviews have synthesized the evidence comparing TAP blocks with local anesthetic would infiltration [52-54]. The evidence from these reviews indicates that TAP blocks provide superior and longer lasting analgesia when compared with local anesthetic wound infiltration. 


\section{TAP Blocks in Different Surgical Populations}

\section{Cesarean Delivery}

Three systematic reviews on TAP blocks in the cesarean delivery population show similar results to the general surgical population $[55,56,57 \bullet \bullet$. The reviews consistently show analgesic benefit of TAP blocks in patients who did not receive intrathecal morphine. No analgesic benefit of TAP block was observed when intrathecal morphine was used. Intrathecal morphine was associated with longer duration of analgesia compared with TAP block alone at the expense of an increased incidence of side effects such as pruritus [57••].

A recent review concluded that low-dose TAP blocks (bupivacaine equivalents less than or equal to $50 \mathrm{mg}$ per side) for cesarean delivery provide analgesia and opioid-sparing effects comparable with high-dose TAP blocks. Therefore, a lower dose TAP block should be used to reduce the risk of local anesthetic toxicity without compromising the analgesic efficacy [58]. Another systematic review comparing TAP blocks with wound infiltration for cesarean delivery demonstrated no statistically significant difference in analgesia or side effects for patients who received multimodal analgesia but no intrathecal morphine [59].

\section{Hysterectomy}

Three recent systematic reviews found that TAP blocks provide significant postoperative early and 24-h pain control compared with placebo or no block among women who undergo a hysterectomy. There was reduced morphine consumption among patients who underwent total abdominal hysterectomy but not for total laparoscopic hysterectomy [60-62].

\section{Pediatric Population}

A recent review synthesized the evidence on rectus sheath and transversus abdominis plane blocks in children. Data from ten RCTs $(n=599)$ suggests that patients receiving TAP and RSB have a small but significant reduction in pain score and opioid use $6-8 \mathrm{~h}$ after surgery. However, a high level of heterogeneity in the included studies warrant caution in the interpretation of results [7].

\section{Dexamethasone as an Adjuvant}

Two recent systematic reviews have shown that dexamethasone added to local anesthetics in ultrasoundguided TAP block significantly decreased visual analogue pain scores by 1 point on a scale of $0-10$ at rest for first $12 \mathrm{~h}$, prolonged the time to the first request for additional analgesics by $3 \mathrm{~h}$, and reduced the incidence of postoperative nausea and vomiting significantly (risk ratios =
$0.40)$ without any increase in reported complications compared with the control group [63, 64••].

\section{Dexmedetomidine as an Adjuvant}

Another recent review that included twenty published trials found that the addition of dexmedetomidine significantly reduced rest pain scores for $8 \mathrm{~h}$ postoperatively (mean difference $0.8 / 10$ ) and $4 \mathrm{~h}$ postoperatively on movement. Adjuvant dexmedetomidine significantly prolonged the duration of the TAP block by $3.3 \mathrm{~h}$ and did not affect the incidence of postoperative nausea and vomiting, hypotension, bradycardia, somnolence, or pruritus [65].

\section{Liposomal Bupivacaine}

Currently, there is a lack of data to support or refute the use of liposomal bupivacaine in TAP blocks for the management of postoperative pain [66].

\section{Continuous TAP Catheters}

A recent systematic review on TAP catheters included twelve small randomized controlled trials. The studies were extremely heterogeneous regarding the type of surgery, control group, and technique of catheter insertion and infusion. The authors concluded that TAP catheters have the potential to provide effective and safe analgesia. A few studies have demonstrated effects comparable with TEA, while TAP catheters are shown to be consistently superior to systemic analgesia alone. Common complications of TAP catheters include catheter dislodgement, block failures, and catheter leakage [67•].

\section{Complications}

It has been shown that the local anesthetic systemic concentrations commonly exceed accepted thresholds after both TAP blocks and RSB [24••]. Although there are case reports of seizures and ventricular arrhythmias, the incidence of local anesthetic systemic toxicity is extremely low. However, efforts should be made to not exceed the maximum recommended milligrams per kilogram local anesthetic dose [68]. This may be accomplished by using a lower concentration of local anesthetic as $0.375 \%$ ropivacaine and $0.5 \%$ ropivacaine have been shown to be equally effective for TAP block [69]. The risk of hematoma or visceral damage is extremely rare with ultrasound guidance. Femoral nerve block has been reported after TAP block, leading to 6-8 h of quadriceps weakness especially with larger volume of injectate [70]. 


\section{Conclusion}

Ultrasound-guided TAP block provide $12-24 \mathrm{~h}$ of postoperative analgesic benefit after abdominal laparotomy, laparoscopy, and cesarean delivery when long-acting neuraxial opioids are not used or contraindicated. It appears to be a safe technique, especially with ultrasound guidance. Consideration should be given to adding an adjuvant to prolong its duration of action and improve efficacy of single-shot TAP blocks. Although continuous TAP catheters have been used, they require maintenance of multiple catheter infusions and risk of failure. The studies evaluating its impact on the long-term and functional outcomes are lacking.

\section{Compliance with Ethical Standards}

Conflict of Interest Vishal Uppal, Sushil Sancheti, and Hari Kalagara declare that he has no conflict of interest.

Human and Animal Rights and Informed Consent This article does not contain any studies with human or animal subjects performed by any of the authors.

Open Access This article is distributed under the terms of the Creative Commons Attribution 4.0 International License (http:// creativecommons.org/licenses/by/4.0/), which permits unrestricted use, distribution, and reproduction in any medium, provided you give appropriate credit to the original author(s) and the source, provide a link to the Creative Commons license, and indicate if changes were made.

\section{References}

Papers of particular interest, published recently, have been highlighted as:

- Of importance

•• Of major importance

1. Willschke H, Bosenberg A, Marhofer P, et al. Ultrasonographyguided rectus sheath block in paediatric anaesthesia-a new approach to an old technique. British Journal of Anaesthesia. 2006;97:244-9.

2. Dolan J, Lucie P, Geary T, Smith M, Kenny GN. The rectus sheath block: accuracy of local anesthetic placement by trainee anesthesiologists using loss of resistance or ultrasound guidance. Regional Anesthesia and Pain Medicine. 2009;34:247-50.

3. Xu L, Hu Z, Shen J, McQuillan PM. Efficacy of US-guided transversus abdominis plane block and rectus sheath block with ropivacaine and dexmedetomidine in elderly high-risk patients. Minerva Anestesiologica. 2018;84:18-24.

4. Landmann A, Visoiu M, Malek MM. Development of a novel technique for bilateral rectus sheath nerve blocks under laparoscopicguidance. Journal of Pediatric Surgery. 2017;52:966-9.

5. Purdy M, Kinnunen M, Kokki M, et al. A prospective, randomized, open label, controlled study investigating the efficiency and safety of 3 different methods of rectus sheath block analgesia following midline laparotomy. Medicine. 2018;97:e9968.

6. Kartalov A, Jankulovski N, Kuzmanovska B, et al. The effect of rectus sheath block as a supplement of general anesthesia on postoperative analgesia in adult patient undergoing umbilical hernia repair. Pril (Makedon Akad Nauk Umet Odd Med Nauki). 2017;38:135-42.

7.• Hamill JK, Rahiri JL, Liley A, Hill AG. Rectus sheath and transversus abdominis plane blocks in children: a systematic review and meta-analysis of randomized trials. Paediatric Anaesthesia. 2016;26:363-71 A meta-analysis of RCTs in children with RSB and TAP blocks in abdominal operations. Abdominal wall blocks reduce pain and opiate use in children.

8. Bashandy GM, Elkholy AH. Reducing postoperative opioid consumption by adding an ultrasound-guided rectus sheath block to multimodal analgesia for abdominal cancer surgery with midline incision. Anesthesiology and Pain Medicine. 2014;4:e18263.

9. Elbahrawy K, El-Deeb A. Rectus sheath block for postoperative analgesia in patients with mesenteric vascular occlusion undergoing laparotomy: a randomized single-blinded study. Anesthesia, Essays and Researches. 2016;10:516-20.

10. Hong S, Kim H, Park J. Analgesic effectiveness of rectus sheath block during open gastrectomy: a prospective double-blinded randomized controlled clinical trial. Medicine. 2019;98:e15159.

11. Lovkov IA, Uvarov DN, Antipin EE, Ushakov AA, Karpunov AA, Nedashkovskiv EV. Efficiency and safety of bilateral ultrasound rectus sheath block in urgent laparotomy. Anesteziologiia i Reanimatologiia. 2017;62:60-3.

12. Abdelsalam K, Mohamdin OW. Ultrasound-guided rectus sheath and transversus abdominis plane blocks for perioperative analgesia in upper abdominal surgery: a randomized controlled study. Saudi Journal of Anaesthesia. 2016;10:25-8.

13. Yassen K, Lotfy M, Miligi A, Sallam A, Hegazi EAR, Afifi M. Patient-controlled analgesia with and without transverse abdominis plane and rectus sheath space block in cirrhotic patients undergoing liver resection. Journal of Anaesthesiology, Clinical Pharmacology. 2019;35:58-64.

14. Bakshi SG, Mapari A, Shylasree TS. REctus Sheath block for postoperative analgesia in gynecological ONcology Surgery (RESONS): a randomized-controlled trial. Canadian Journal of Anaesthesia = Journal Canadien D'anesthesie. 2016;63:1335-44 An RCT evaluating the efficacy of bilateral RCB catheters for postoperative midline laparotomy analgesia. The study demonstrates the benefits of RCB catheters for opioid reduction up to $48 \mathrm{~h}$.

15. Breschan C, Jost R, Stettner H, et al. Ultrasound-guided rectus sheath block for pyloromyotomy in infants: a retrospective analysis of a case series. Paediatric Anaesthesia. 2013;23:1199-204.

16. Karaarslan E, Topal A, Avci O, Tuncer US. Research on the efficacy of the rectus sheath block method. Agri : Agri (Algoloji) Dernegi'nin Yayin organidir $=$. The Journal of the Turkish Society of Algology. 2018;30:183-8.

17. Hausken J, Rydenfelt K, Horneland R, et al. First experience with rectus sheath block for postoperative analgesia after pancreas transplant: a retrospective observational study. Transplantation Proceedings. 2019;51:479-84.

18. Tudor EC, Yang W, Brown R, Mackey PM. Rectus sheath catheters provide equivalent analgesia to epidurals following laparotomy for colorectal surgery. Annals of the Royal College of Surgeons of England. 2015;97:530-3.

19. Chung W, Yoon Y, Kim JW, et al. Comparing two different techniques of rectus sheath block after single port laparoscopic surgery in benign adnexal mass patients: Surgical versus ultrasonography guidance-a randomized, single-blind, case-controlled study. European Journal of Obstetrics, Gynecology, and Reproductive Biology. 2017;217:29-33. 
20. Cho S, Kim YJ, Jeong K, Moon HS. Ultrasound-guided bilateral rectus sheath block reduces early postoperative pain after laparoscopic gynecologic surgery: a randomized study. Journal of Anesthesia. 2018;32:189-97 An RCT evaluating the analgesic efficacy of ultrasound-guided bilateral RCB after laparoscopic surgery. US-BRSB reduces immediate postoperative pain and opioid consumption.

21. Gupta M, Naithani U, Singariya G. Gupta S Comparison of $0.25 \%$ ropivacaine for intraperitoneal instillation $\mathrm{v} / \mathrm{s}$ rectus sheath block for postoperative pain relief following laparoscopic cholecystectomy: a prospective study. J Clin Diagn Res. 2016;10:UC10-5.

22. Hamill JK, Liley A, Hill AG. Rectus sheath block for laparoscopic appendicectomy: a randomized clinical trial. ANZ Journal of Surgery. 2015;85:951-6.

23. Maloney C, Kallis M, El-Shafy IA, Lipskar AM, Hagen J, Kars M. Ultrasound-guided bilateral rectus sheath block vs. conventional local analgesia in single port laparoscopic appendectomy for children with nonperforated appendicitis. Journal of Pediatric Surgery. 2018;53:431-6.

24.• Rahiri J, Tuhoe J, Svirskis D, Lightfoot NJ, Lirk PB, Hill AG. Systematic review of the systemic concentrations of local anaesthetic after transversus abdominis plane block and rectus sheath block. British Journal of Anaesthesia. 2017;118:517-26 A systematic review evaluating systemic concentrations of LA after TAP and RSB and the risk of systemic toxicity. The study concludes that the LA in TAP and RSB can lead to detectable systemic concentrations but relatively safe with regard to LA systemic toxicity.

25. Kitayama M, Wada M, Hashimoto H, Kudo T, Takada N, Hirota K. Effects of adding epinephrine on the early systemic absorption kinetics of local anesthetics in abdominal truncal blocks. Journal of Anesthesia. 2014;28:631-4.

26. Hebbard P, Fujiwara Y, Shibata Y, Royse C. Ultrasound-guided transversus abdominis plane (TAP) block. Anaesthesia and Intensive Care. 2007;35:616-7.

27. Yoshiyama S, Ueshima H, Sakai R, Otake HA. Posterior TAP block provides more effective analgesia than a lateral TAP block in patients undergoing laparoscopic gynecologic surgery: a retrospective study. Anesthesiology Research and Practice. 2016;2016:4598583.

28. Hebbard P. Subcostal transversus abdominis plane block under ultrasound guidance. Anesthesia and Analgesia. 2008;106:674-5 author reply 5 .

29. Niraj G, Kelkar A, Hart E, et al. Comparison of analgesic efficacy of four-quadrant transversus abdominis plane (TAP) block and continuous posterior TAP analgesia with epidural analgesia in patients undergoing laparoscopic colorectal surgery: an open-label, randomised, non-inferiority trial. Anaesthesia. 2014;69:348-55.

30. Abdallah FW, Laffey JG, Halpern SH, Brull R. Duration of analgesic effectiveness after the posterior and lateral transversus abdominis plane block techniques for transverse lower abdominal incisions: a meta-analysis. British Journal of Anaesthesia. 2013;111:721-35.

31. Ganapathy S, Sondekoppam RV, Terlecki M, Brookes J, Das Adhikary S, Subramanian L. Comparison of efficacy and safety of lateral-to-medial continuous transversus abdominis plane block with thoracic epidural analgesia in patients undergoing abdominal surgery: a randomised, open-label feasibility study. European Journal of Anaesthesiology. 2015;32:797-804.

32. Ma N, Duncan JK, Scarfe AJ, Schuhmann S, Cameron AL. Clinical safety and effectiveness of transversus abdominis plane (TAP) block in post-operative analgesia: a systematic review and metaanalysis. Journal of Anesthesia. 2017;31:432-52.

33.• Brogi E, Kazan R, Cyr S, Giunta F, Hemmerling TM. Transversus abdominal plane block for postoperative analgesia: a systematic review and meta-analysis of randomized-controlled trials. Canadian Journal of Anaesthesia = Journal Canadien
D'anesthesie. 2016;63:1184-96 A meta-analysis of TAP blocks for postoperative analgesia with inclusion of 51 trials with different control groups. The TAP block can play an important role by reducing postoperative pain scores and 24-h morphine consumption.

34. Baeriswyl M, Kirkham KR, Kern C, Albrecht E. The analgesic efficacy of ultrasound-guided transversus abdominis plane block in adult patients: a meta-analysis. Anesthesia and Analgesia. 2015;121:1640-54.

35. Ripolles J, Mezquita SM, Abad A, Calvo J. Analgesic efficacy of the ultrasound-guided blockade of the transversus abdominis plane - a systematic review. Brazilian Journal of Anesthesiology (Elsevier). 2015;65:255-80.

36. Johns N, O'Neill S, Ventham NT, Barron F, Brady RR, Daniel T. Clinical effectiveness of transversus abdominis plane (TAP) block in abdominal surgery: a systematic review and meta-analysis. Colorectal Disease : the official journal of the Association of Coloproctology of Great Britain and Ireland. 2012;14:e635-42.

37. Siddiqui MRS, Sajid MS, Baig MK. Clinical effectiveness of the transversus abdominis plane (TAP) block in patients undergoing abdominal surgery: a systematic review. Colorectal Disease. 2009;11:38.

38. Liu L, Xie YH, Zhang W, Chai XQ. Effect of transversus abdominis plane block on postoperative pain after colorectal surgery: a metaanalysis of randomized controlled trials. Medical Principles and Practice : International Journal of the Kuwait University, Health Science Centre. 2018;27:158-65.

39. Hain E, Maggiori L, Prost A, la Denise J, Panis Y. Transversus abdominis plane (TAP) block in laparoscopic colorectal surgery improves postoperative pain management: a meta-analysis. Colorectal Disease : the official journal of the Association of Coloproctology of Great Britain and Ireland. 2018;20:279-87.

40. Hain E, Maggiori L, Denise JP, Panis Y. Transversus abdominis plane (TAP) block in laparoscopic colorectal surgery: a meta-analysis. Colorectal Disease. 2016;18:18 This meta-analysis aimed to assess the efficacy of TAP block after laparoscopic colorectal surgery. TAP block in laparoscopic colorectal surgery improves postoperative opioid consumption and recovery of postoperative digestive function.

41. Oh TK, Lee SJ, Do SH, Song IA. Transversus abdominis plane block using a short-acting local anesthetic for postoperative pain after laparoscopic colorectal surgery: a systematic review and metaanalysis. Surgical Endoscopy. 2018;32:545-52.

42. Zhao X, Tong Y, Ren H, et al. Transversus abdominis plane block for postoperative analgesia after laparoscopic surgery: a systematic review and meta-analysis. International Journal of Clinical and Experimental Medicine. 2014;7:2966-75.

43. De Oliveira GS Jr, Castro-Alves LJ, Nader A, Kendall MC, McCarthy RJ. Transversus abdominis plane block to ameliorate postoperative pain outcomes after laparoscopic surgery: a metaanalysis of randomized controlled trials. Anesthesia and Analgesia. 2014;118:454-63.

44. Peng K, Ji FH, Liu HY. Wu SR Ultrasound-guided transversus abdominis plane block for analgesia in laparoscopic cholecystectomy: a systematic review and meta-analysis. Medical Principles and Practice : International Journal of the Kuwait University, Health Science Centre. 2016;25:237-46.

45. Ni X, Zhao X, Li M, Li Q, Liu Z. The effect of transversus abdominis plane block for pain after laparoscopic cholecystectomy: a meta-analysis of randomized controlled trials. International Journal of Clinical and Experimental Medicine. 2016;9:9974-82.

46. Gao T, Zhang JJ, Xi FC, et al. Evaluation of transversus abdominis plane (TAP) block in hernia surgery: a meta-analysis. The Clinical Journal of Pain. 2017;33:369-75.

47. Singh PM, Borle A, Makkar JK, Trisha A, Sinha A. Evaluation of transversus abdominis plane block for renal transplant recipients - a 
meta-analysis and trial sequential analysis of published studies. Saudi Journal of Anaesthesia. 2018;12:261-71.

48. Abdallah FW, Brull R. Transversus abdominis plane block: can TAP block survive the challenge of multimodal analgesia? A systematic review. Regional Anesthesia and Pain Medicine. 2012;37.

49. Kong G, Bravos ED, Eng C, Mathur V, Wu C. Meta-analysis of the efficacy of transversus abdominis plane block after abdominal surgery. Regional Anesthesia and Pain Medicine. 2010;35.

50. Charlton S, Cyna AM, Middleton P, Griffiths JD. Perioperative transversus abdominis plane (TAP) blocks for analgesia after abdominal surgery. Cochrane Database Syst Rev. 2010;(12): CD007705.

51. Baeriswyl M, Zeiter F, Piubellini D, Kirkham KR, Albrecht E. The analgesic efficacy of transverse abdominis plane block versus epidural analgesia: a systematic review with meta-analysis. Medicine. 2018;97:e11261 This meta-analysis aimed to compare the analgesic efficacy of epidural analgesia and TAP block. There is moderate evidence that TAP block and epidural analgesia are equally effective in treating postoperative pain, while TAP block is associated with fewer side effects.

52. Guo Q, Li R, Wang L, Zhang D, Ma Y. Transversus abdominis plane block versus local anaesthetic wound infiltration for postoperative analgesia: a systematic review and meta-analysis. International Journal of Clinical and Experimental Medicine. 2015;8:17343-52.

53. Yu N, Long X, Lujan-Hernandez JR, Succar J, Xin X, Wang X. Transversus abdominis-plane block versus local anesthetic wound infiltration in lower abdominal surgery: a systematic review and meta-analysis of randomized controlled trials. BMC Anesthesiology. 2014;14:121-2253-14-121 eCollection 014.

54. Ventham NT, O'Neill S, Johns N, Brady RR, Fearon KC. Evaluation of novel local anesthetic wound infiltration techniques for postoperative pain following colorectal resection surgery: a meta-analysis. Diseases of the Colon and Rectum. 2014;57:237-50.

55. Fusco P, Scimia P, Paladini G, et al. Transversus abdominis plane block for analgesia after Cesarean delivery. A systematic review. Minerva Anestesiologica. 2015;81:195-204.

56. Singh SI, Kumar K, Kolesnichenko AP, Jones P. The analgesic efficacy of tap blocks after cesarean deliveries: a systematic review. Canadian Journal of Anesthesia. 2012;59.

57.• Mishriky BM, George RB, Habib AS. Transversus abdominis plane block for analgesia after cesarean delivery: a systematic review and meta-analysis. Canadian journal of anaesthesia $=$ Journal canadien d'anesthesie. 2012;59:766-78 A meta-analysis to assess the analgesic efficacy of TAP block for cesarean delivery. TAP block significantly improved postoperative analgesia in women undergoing cesarean delivery who did not receive intrathecal morphine but showed no improvement in those who received intrathecal morphine.

58. Ng SC, Habib AS, Sodha S, Carvalho B, Sultan P. High-dose versus low-dose local anaesthetic for transversus abdominis plane block post-caesarean delivery analgesia: a meta-analysis. British Journal of Anaesthesia. 2018;120:252-63.

59. Patel SD, Halpern S, Jadin S, Howlef R, Carvalho B. Sultan P Comparing TAP block and wound infiltration for postoperative analgesia following cesarean delivery: a meta-analysis. International Journal of Obstetric Anesthesia. 2018;35:S8.

60. Bacal V, Rana U, McIsaac DI, Chen I. Transversus abdominis plane block for post hysterectomy pain: a systematic review and meta-analysis. Journal of Minimally Invasive Gynecology. 2019;26:40-52.

61. Tubog TD, Harenberg JL, Mason-Nguyen J, Kane TD. Opioidsparing effects of transversus abdominis plane block in elective hysterectomy: a systematic review and meta-analysis. AANA Journal. 2018;86:41-55.

62. Wang A. Shin JH Effectiveness of the transversus abdominis plane (TAP) block in laparoscopic and robotic gynecologic surgery. Journal of Minimally Invasive Gynecology. 2017;24:S11.

63. Zhang D, Zhou C, Wei D, Ge L. Li Q Dexamethasone added to local anesthetics in ultrasound-guided transversus abdominis plain (TAP) block for analgesia after abdominal surgery: a systematic review and meta-analysis of randomized controlled trials. PloS One. 2019;14:e0209646.

64.• Chen Q, An R, Zhou J, Yang B. Clinical analgesic efficacy of dexamethasone as a local anesthetic adjuvant for transversus abdominis plane (TAP) block: a meta-analysis. PloS One. 2018;13:e198923 This meta-analysis sought to assess the efficacy of dexamethasone in prolonging the TAP block analgesia. Dexamethasone prolongs the LA effect when used as an adjuvant in TAP block and improves the analgesic effects of the block.

65. Sun Q, Liu S, Wu H, et al. Dexmedetomidine as an adjuvant to local anesthetics in transversus abdominis plane block: a systematic review and meta-analysis. The Clinical Journal of Pain. 2019;35:37584.

66. Hamilton TW, Athanassoglou V, Trivella M et al. Liposomal bupivacaine peripheral nerve block for the management of postoperative pain. Cochrane Database Syst Rev. 2016;(8):CD011476.

67. Sanderson BJ, Doane MA. Transversus abdominis plane catheters for analgesia following abdominal surgery in adults. Regional Anesthesia and Pain Medicine. 2018;43:5-13 This systematic review is to evaluate the efficacy and safety of TAP catheters for postoperative analgesia following abdominal surgery in adults. There are promising indications for the benefit of TAP catheters when there is more standardization of patient care.

68. Scherrer V, Compere V, Loisel C, Dureuil BJA. Practice A Cardiac arrest from local anesthetic toxicity after a field block and transversus abdominis plane block: a consequence of miscommunication between the anesthesiologist and surgeon. A A Case Rep. 2013;1:75-6.

69. Sun N, Wang S, Ma P, Liu S, Shao A, Xiong L. Postoperative analgesia by a transversus abdominis plane block using different concentrations of ropivacaine for abdominal surgery: a meta-analysis. The Clinical Journal of Pain. 2017;33:853-63.

70. Manatakis DK, Stamos N, Agalianos C, Karvelis MA, Gkiaourakis M. Davides D Transient femoral nerve palsy complicating "blind" transversus abdominis plane block. Case Rep Anesthesiol. 2013;2013:874215.

Publisher's Note Springer Nature remains neutral with regard to jurisdictional claims in published maps and institutional affiliations. 